\title{
Stiffness and Temporal Optimization in Periodic Movements: An Optimal Control Approach
}

\author{
Jun Nakanishi, Konrad Rawlik and Sethu Vijayakumar
}

\begin{abstract}
We present a novel framework for stiffness and temporal optimization of periodic movements, with an emphasis on exploiting the intrinsic passive dynamics to realize efficient actuation and control. We use a dynamical systems based representation tuned to the requirements of rhythmic movements and propose a systematic methodology to optimize for control commands, temporal aspect of movements and timevarying stiffness profiles from first principles of optimality. Evaluations on a single pendulum and underactuated two-link robot simulation highlight the benefits, achieving remarkable actuation efficiency on complicated, highly dynamic tasks such as swing-up and brachiation.
\end{abstract}

\section{INTRODUCTION}

Optimality principles have been drawing much attention in motor control of biological and artificial systems. Optimal control theory has been used as a theoretical model of movement generation in biological motor control [1]. From a practical point of view, there is growing interest in the design and control of robotic systems using variable stiffness mechanisms with the aim of achieving compliant and energy efficient movement, including methods that use optimal control approaches [2].

Periodic movements are an interesting family of movements, with examples such as walking and running, that make maximally effective use of the intrinsic dynamics of the system. However, to our knowledge, while there have been a number of previous studies on optimal control of discrete movements such as point-to-point reaching tasks, there is a limited amount of work on the optimal control of periodic movements; relevant studies include optimization of biped locomotion using differential dynamic programming (DDP) [3], Poincaré map based optimization of biped locomotion [4], [5], [6], and stabilization of periodic systems and movements [7], [8], respectively, from a control theoretic point of view.

In this paper, we present an approach to stiffness and temporal optimization in periodic movements with a focus on exploiting the intrinsic dynamics of the system. Dynamical systems based state-space representations of periodic movements have significant merits over time indexed representations [9] - some key aspects include ease of frequency, amplitude and offset modulation while providing robustness to perturbations during control. We formulate a phase oscillator based dynamical system representation that maintains all the key benefits while using Fourier basis functions instead of Gaussian basis functions [10], primarily because frequency space representations are more appropriate for periodic trajectories, i.e., Fourier basis functions

J. Nakanishi, K. Rawlik and S. Vijayakumar are with the Institute of Perception, Action and Behaviour, University of Edinburgh, Edinburgh EH8 9AB, UK. Email: \{jun.nakanishi, K.C.Rawlik, sethu.vijayakumar\}@ed.ac.uk form orthonormal bases. Given an appropriate representation of periodic movements, we formulate an appropriate cost function and employ an optimal feedback control framework [11] to compute an optimal sequence of driving commands.

In addition to obtaining an optimal control law, we are interested in optimizing the temporal aspect of the movement pattern itself in order to exploit the intrinsic passive dynamics of the system and achieve energy efficient control. Specifically, we consider the problem of finding the optimal speed of the periodic movement, i.e., the frequency or period of the pattern. This problem is motivated by the observation that, for example, the 'natural' walking cycle appears to be closely coupled to the dimensions and mass properties of the limbs, i.e., it should have some relationship with the intrinsic dynamics to achieve energy efficient behavior. In order to exploit this insight, we extend our time-optimal control framework proposed in [12] to optimize the temporal aspect of periodic movements.

When executing periodic movements, there is strong evidence of time-varying stiffness profiles in biological systems, e.g., human elbow joint stiffness modulation during cyclic movement [14]. Optimization of stiffness for periodic movements has been considered by a few studies, e.g., [15], [16]. However, in the majority of cases, constant stiffness is assumed during one period of movement. In this paper, we would like to go beyond the minimization of the stiffness norm approach employed in reaching and via-point tasks [17] and optimize for temporal stiffness modulation by exploiting the target periodicity and plant dynamics. We evaluate our proposed approach in numerical studies using realistic, fullphysics dynamics. Simulation results demonstrate that it is possible to achieve energy efficient control of periodic movements by exploiting the intrinsic dynamics of the system as a result of optimization.

\section{Problem Formulation}

\section{A. Representation of Periodic Movement}

We start by considering an appropriate representation for rhythmic movement trajectories. A time-indexed Fourier series expansion with $N$ harmonics can be written as

$$
y(t)=a_{0}+\sum_{n=1}^{N}\left(a_{n} \cos \frac{2 n \pi t}{T}+b_{n} \sin \frac{2 n \pi t}{T}\right)
$$

where $T$ is the period of oscillation, and $a_{n}$ and $b_{n}$ are Fourier coefficients. These coefficients can be computed efficiently using a method presented in [18] to fit any given sampled periodic trajectory. The number of harmonics $N$ controls the complexity of the trajectory. In this paper, instead of using the time-indexed representation above, we suggest the following reformulation of (1) in terms of an 

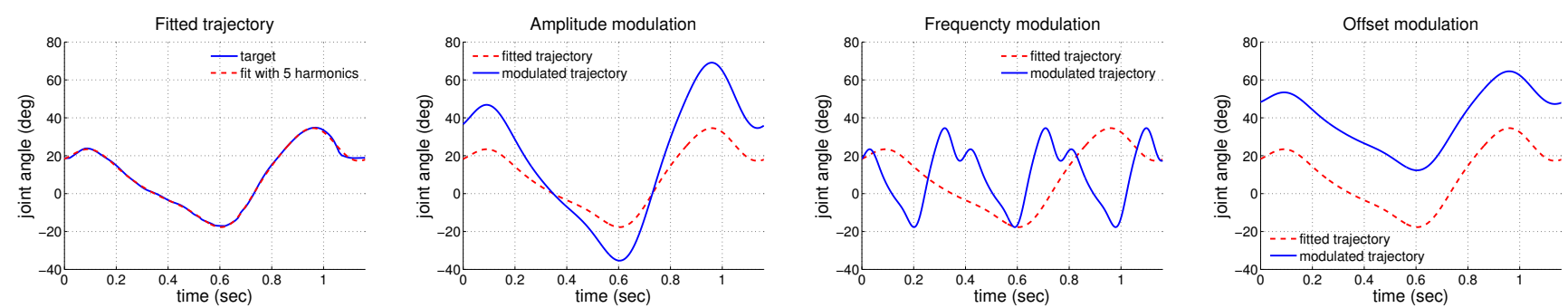

Fig. 1. Fitting and modulation of hip joint trajectory of human walking data taken from [13] using (2) and (3). Left: original trajectory and fitting using 5 harmonics. Second: Amplitude modulation with $r=2$. Third: Frequency modulation with $3 \omega$. Right: Offset modulation with $y_{\text {off }}=30$.

autonomous dynamical system including phase oscillator dynamics $\phi$ :

$$
\begin{aligned}
y(t) & =r \boldsymbol{\psi}^{T}(\phi) \boldsymbol{\theta}+y_{\text {off }} \\
\dot{\phi} & =\omega
\end{aligned}
$$

where

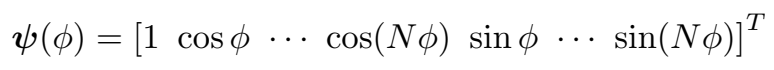

is the Fourier basis function vector,

$$
\boldsymbol{\theta}=\left[\begin{array}{lllllll}
a_{0} & a_{1} & \cdots & a_{N} & b_{1} & \cdots & b_{N}
\end{array}\right]^{T}
$$

is the parameter vector composed of Fourier coefficients, $y$ is the trajectory, $\phi$ is the phase, $\omega$ is the angular frequency $\left(\omega=\frac{2 \pi}{T}\right), r$ is the amplitude, and $y_{\text {off }}$ is the offset of the trajectory. With such a formulation, as previously seen with Dynamic Movement Primitives (DMPs) [10], it is possible to easily scale the frequency, amplitude and offset of the reference trajectory and potentially incorporate additional coupling with external signals. As an illustrative example, in Fig. 1, we show the fitting and modulation of a hip joint trajectory of human walking taken from [13] using the representation of periodic trajectories introduced here in (2) and (3). The period of the trajectory is $T=1.167 \mathrm{sec}$ and $N=5$ harmonics are used. The left plot in Fig. 1 shows the fitting result. Note that the accuracy of fitting can be improved by increasing the number of harmonics $N$. The next three sub-plots show examples of amplitude, frequency and offset modulation by changing $r, \omega$ and $y_{\text {off }}$, respectively, in (2) and (3).

It is important to note that the Fourier series expansion based representation introduced here includes Lissajous figures [19] which can produce a large number of interesting complex periodic trajectories, allowing for a rich movement repertoire. In the following, we use the output $y(t)$ given by (2) as a reference (desired) movement trajectory as defined in (6) below.

\section{B. Optimal Feedback Control for Rhythmic Tasks}

Next we consider the optimization of control commands for a periodic trajectory tracking task with a given fixed frequency $\omega$. For plant dynamics

$$
\dot{\mathbf{x}}=\mathbf{f}(\mathbf{x}, \mathbf{u}),
$$

consider the task of tracking a desired periodic trajectory

$$
\mathbf{x}_{r e f}(t)=[y, \dot{y}]^{T}
$$

given by the output of (2) while reducing the control effort u. Note that $\dot{y}$ can be obtained by analytical time derivative of (2). We use the cost function

$$
J=\Phi(\mathbf{x}(0), \mathbf{x}(T))+\int_{0}^{T} h(\mathbf{x}(t), \mathbf{u}(t)) d t .
$$

where $h(\mathbf{x}(t), \mathbf{u}(t))$ is the running cost defined as

$$
h(\mathbf{x}(t), \mathbf{u}(t))=\left(\mathbf{x}-\mathbf{x}_{r e f}\right)^{T} \mathbf{Q}\left(\mathbf{x}-\mathbf{x}_{r e f}\right)+\mathbf{u}^{T} \mathbf{R} \mathbf{u},
$$

$\mathbf{Q}$ and $\mathbf{R}$ are positive (semi)definite matrices and $\Phi(\mathbf{x}(0), \mathbf{x}(T))$ is the terminal cost defined as

$$
\Phi(\mathbf{x}(0), \mathbf{x}(T))=(\mathbf{x}(T)-\mathbf{x}(0))^{T} \mathbf{P}_{T}(\mathbf{x}(T)-\mathbf{x}(0))
$$

where $\mathbf{P}_{T}$ is a positive definite matrix and $T$ is the duration of the movement (i.e., period of the trajectory). Note that the terminal cost $\Phi$ is introduced to enforce the periodicity of the resultant trajectory by minimizing the difference between the states at the beginning and end of the periodic trajectory as motivated in [3]. However, since the criterion in the terminal cost (9) is redundant when the tracking error criterion is explicitly specified in the running cost (8), the term (9) can be removed or have small weight in practice.

Since solving general nonlinear optimal control problems is not analytically tractable, we employ the Iterative Linear Quadratic Gaussian (ILQG) algorithm [11] to obtain a locally optimal feedback control law

$$
\mathbf{u}(\mathbf{x}, t)=\mathbf{u}^{o p t}(t)+\mathbf{L}(t)\left(\mathbf{x}(t)-\mathbf{x}^{o p t}(t)\right)
$$

where $\mathbf{u}^{\text {opt }}(t)$ is the feedforward optimal control sequence, $\mathbf{L}(t)$ is the optimal feedback gain matrix, and $\mathbf{x}^{\text {opt }}(t)$ is the locally optimal trajectory. ILQG [11] is one of a number of iterative methods to approximately solve such nonlinear optimal control problems and has been widely applied to point-to-point and via-point reaching tasks, e.g., in biological [20] and robotic [2] motor control.

The standard rigid body dynamics (RBD) of a movement system including viscous friction is given by

$$
\mathbf{M}(\mathbf{q}) \ddot{\mathbf{q}}+\mathbf{C}(\mathbf{q}, \dot{\mathbf{q}}) \dot{\mathbf{q}}+\mathbf{g}(\mathbf{q})+\mathbf{D} \dot{\mathbf{q}}=\boldsymbol{\tau}
$$

where $\mathbf{q}$ is the joint angle vector, $\mathbf{M}$ is the inertia matrix, $\mathbf{C}$ is the Coriolis term, $\mathbf{g}$ is the gravity vector, $\mathbf{D}$ is the viscous damping matrix, and $\tau$ is the joint torque vector. Therefore, the plant dynamics given in (5) takes the specific form

$$
\mathbf{f}=\left[\begin{array}{c}
\mathbf{x}_{2} \\
\mathbf{M}^{-1}\left(\mathbf{x}_{1}\right)\left(-\mathbf{C}\left(\mathbf{x}_{1}, \mathbf{x}_{2}\right) \mathbf{x}_{2}-\mathbf{g}\left(\mathbf{x}_{1}\right)-\mathbf{D} \dot{\mathbf{x}}_{2}+\mathbf{u}\right)
\end{array}\right]
$$

where $\mathbf{x}=\left[\begin{array}{ll}\mathbf{x}_{1}^{T}, & \mathbf{x}_{2}^{T}\end{array}\right]^{T}=\left[\begin{array}{ll}\mathbf{q}^{T}, & \dot{\mathbf{q}}^{T}\end{array}\right]^{T}$ and $\mathbf{u}=$ $\tau$. The cost function described above is formulated as a 
trajectory tracking problem. However, note that the suggested formulation in this paper can also be interpreted as finding an optimal control law $\mathbf{u}$ for the augmented autonomous dynamical system

$$
\begin{aligned}
\dot{\mathbf{x}} & =\mathbf{f}(\mathbf{x}, \mathbf{u}) \\
y & =r \boldsymbol{\psi}^{T}(\phi) \boldsymbol{\theta}+y_{o f f} \\
\dot{\phi} & =\omega \\
\mathbf{z} & =\mathbf{x}-\mathbf{y}, \text { where } \mathbf{y}=[y, \dot{y}]
\end{aligned}
$$

such that the plant dynamics (12) would behave like the target dynamics given in (13) and (14) with the running cost

$$
h(\mathbf{z}, \mathbf{u})=\mathbf{z}^{T} \mathbf{Q} \mathbf{z}+\mathbf{u}^{T} \mathbf{R u}
$$

in the cost function (7). By doing this, it is also possible to formulate an on-line optimization based on a receding horizon approach when external coupling is incorporated to the phase dynamics to modulate the desired movement.

\section{Temporal Optimization of the Periodic Movement}

The optimal control formulation above computes an optimal feedback control law (10) for the reference trajectory given in (3) with a fixed frequency $\omega$. However, we are also interested in optimizing the temporal aspect of the periodic movement.

Extending our approach for temporal optimization [12], we define a mapping $\beta(t)$ from the real time $t$ to a canonical time $t^{\prime}$

$$
t^{\prime}=\gamma(t)=\int_{0}^{t} \frac{1}{\beta(s)} d s
$$

where $\beta(\cdot)>0$. Assuming that the $\beta(t)$ is constant during the movement and discretizing (17) yields:

$$
\Delta t^{\prime}=\frac{1}{\beta} \Delta t
$$

By exploiting the fact that the angular frequency and the period of oscillation are related through the total movement duration $T$ as $\omega=\frac{2 \pi}{T}$ and using (18), we can write:

$$
\beta=\frac{\omega^{\prime}}{\omega}=\frac{\Delta t}{\Delta t^{\prime}}
$$

where $\omega^{\prime}$ is the canonical frequency. The mapping $\beta$ is optimized, e.g., using our temporal optimization algorithm [12], to yield an optimized $\omega$.

The optimized $\omega$ is fed back to modify the reference trajectory through (3) and a new set of control signals are computed using the ILQG iterations (Section II-B) until convergence. In many of the rhythmic movement tasks we considered (see Section III-A), we did not have to explicitly introduce a 'time' cost during the temporal optimization, in contrast to discrete movement tasks. The potential explanation is that a movement period closer to the corresponding natural or resonant frequency of the system will require less control effort-reflected in a (local) minimum in the cost function corresponding to an optimal $\omega$.

\section{Time-varying Stiffness Optimization}

Stiffness is the property of a mechanical component characterizing the relationship between the change in the applied force (or torque) and the resultant displacement, and is defined by

$$
\delta \tau=K \delta q
$$

where $K$ is the joint stiffness, $\tau$ is the joint torque and $q$ is the joint angle. For the plant dynamics (11), consider an actuator model with variable stiffness mechanism whose joint torques $\tau$ are given in the form

$$
\boldsymbol{\tau}=\boldsymbol{\tau}(\mathbf{x}, \mathbf{u})=-\mathbf{K}(\mathbf{x}, \mathbf{u})\left(\mathbf{q}-\mathbf{q}_{0}(\mathbf{x}, \mathbf{u})\right)
$$

where $\mathbf{x}=[\mathbf{q}, \dot{\mathbf{q}}]^{T}, \mathbf{q}_{o}$ is the equilibrium position of the joint angles by solving $\boldsymbol{\tau}(\mathbf{x}, \mathbf{u})=\mathbf{0}$ for $\mathbf{q}$ by setting $\boldsymbol{\tau}=\mathbf{0}$ and $\dot{\mathbf{q}}=\mathbf{0}, \mathbf{K}$ is the joint stiffness matrix and $\mathbf{u}$ is the control command [21]. The simplest model of such a variable stiffness actuator can be described by

$$
\boldsymbol{\tau}=-\mathbf{K q}+\mathbf{u}
$$

where the stiffness $\mathbf{K}$ and control inputs $\mathbf{u}$ can be directly and independently controlled. This model can be re-written in the form of (21) by introducing the change of coordinates $\mathbf{u}=\mathbf{K} \mathbf{q}_{0}$. There are several examples of practical mechanical designs of variable stiffness actuators: examples include one that relies on antagonistic actuation [2] or a design with relatively decoupled stiffness and position control [22] (MACCEPA) .

As a toy case, consider a simplified linearized pendulum with a variable stiffness actuator

$$
\ddot{q}+g q=-k q+u \text {. }
$$

The resonant frequency of (23) is given by $\omega=\sqrt{g+k}$ since (23) can be rearranged as

$$
\ddot{q}+(g+k) q=u \text {. }
$$

If the magnitude of the stiffness $k$ and the frequency of the forcing periodic input $\omega$ satisfied the relationship

$$
g+k=\omega^{2}
$$

it would be possible to achieve the desired periodic movement at the frequency of $\omega$ with minimum control effort $u$ by exploiting the resonance property of the oscillatory dynamics. We suggest a generic method for stiffness optimization by using an augmented system dynamics approach as described next.

1) Proposed Formulation: Consider the plant and actuator dynamics with variable stiffness mechanism characterized by k:

$$
\dot{\mathbf{x}}=\mathbf{f}(\mathbf{x}, \mathbf{k}, \mathbf{u})
$$

In the proposed formulation, we augment the plant dynamics, state and commands as

$$
\dot{\mathbf{x}}_{a u g}=\mathbf{f}_{a u g}\left(\mathbf{x}_{a u g}, \mathbf{u}_{a u g}\right)=\left[\begin{array}{c}
\mathbf{f}(\mathbf{x}, \mathbf{k}, \mathbf{u}) \\
\dot{\mathbf{k}}
\end{array}\right]
$$

where

$$
\mathbf{x}_{\text {aug }}=[\mathbf{x}, \mathbf{k}]^{T}, \quad \mathbf{u}_{a u g}=[\mathbf{u}, \dot{\mathbf{k}}]^{T} .
$$

In order to specify an appropriate control cost, we define a quadratic cost of the form $\mathbf{v}_{\text {aug }}^{T} \mathbf{R} \mathbf{v}_{\text {aug }}$ where $\mathbf{v}_{\text {aug }}$ is some 


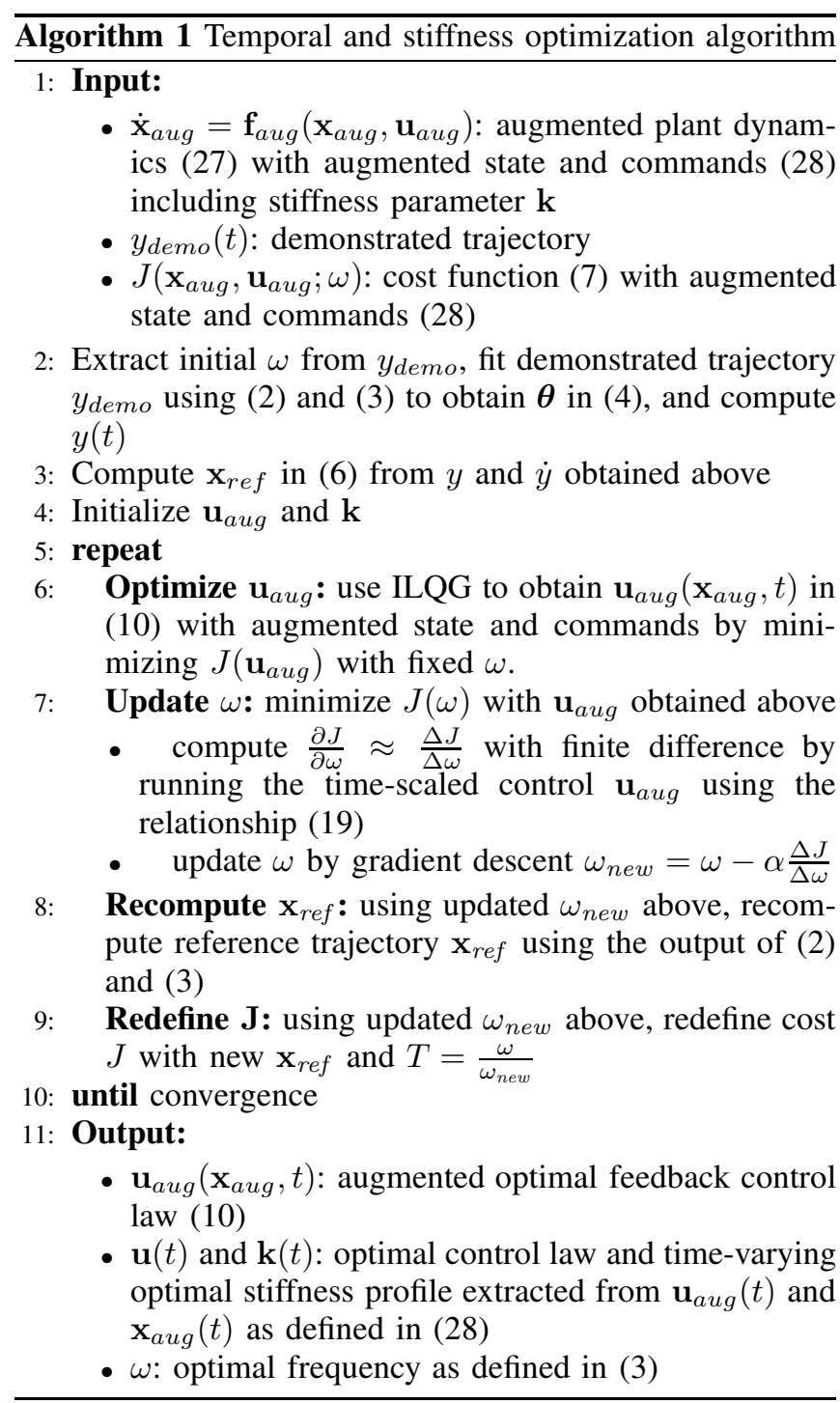

function of $\mathbf{x}_{a u g}$ and $\mathbf{u}_{a u g}$. As a special case of the above, for the actuator model defined in (22), it is sufficient to use $\mathbf{u}_{\text {aug }}^{T} \mathbf{R} \mathbf{u}_{\text {aug }}$ as the running cost. One of the motivations of this formulation is that when not using a penalization of the command and stiffness norm in the cost function (e.g., as used in reaching), the solution of the optimal stiffness is ill-posed. The augmented stiffness velocity term avoids this problem while providing the added benefit of ensuring a (realisable) smooth time-varying stiffness profile. Once we obtain $\mathbf{u}$ and $\mathbf{k}$, it is possible to use the method in [21] to achieve the desired joint angles and stiffness profiles on various practical variable stiffness actuators whose characteristics are given in (21).

The complete temporal and stiffness optimization algorithm is summarized in Algorithm 1.

\section{EVALUATIONS}

\section{A. Single Pendulum}

Under the influence of gravity, mechanical systems exhibit oscillatory movement with a particular frequency depending on the intrinsic dynamics of the system, e.g., a pendulum,

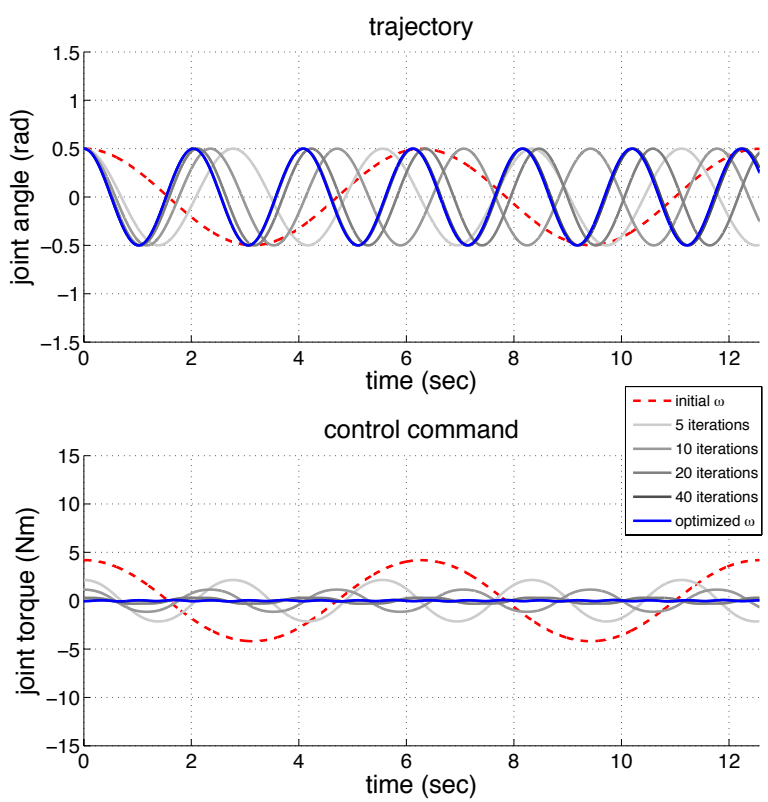

Fig. 2. Results of temporal optimization in a pendulum. Comparison of the joint trajectory $q$ and control input $u$ : initial, during and after optimization of $\omega$. As $\omega$ is updated (converging to near the natural frequency), the magnitude of the required control command is progressively reduced.

or human and robot legs. Consider a single pendulum as an example of an oscillatory system and a special case of (11):

$$
m l^{2} \ddot{q}+d \dot{q}+m g l \sin q=\tau
$$

where $q$ is the joint angle, $m$ is the mass, $l$ is the length, $d$ is the viscous friction coefficient and $g=9.80665$ is the gravitational constant. When the amplitude of the oscillation is small, i.e., $q \ll 1$, the natural frequency and the resonant frequency of the linearized dynamics of (29) are given by $\omega_{0}=\sqrt{\frac{g}{l}}$ in the case of $d=0$.

Fundamentally, if the frequency of the external input $\omega$ matches the natural or resonant frequency $\omega_{0}$, it should be possible to achieve the desired periodic movement while requiring minimal control input.

To test the optimization capability of our method against a benchmark, we consider a pendulum (29) with specific parameters $m=l=1$, which suggests a natural frequency $\omega_{0}=\sqrt{g} \simeq 3.132$ when $q \ll 1$ in the absence of damping. The plant dynamics in the form of (5) can be written as

$$
\dot{\mathbf{x}}=\mathbf{f}(\mathbf{x}, u)=\left[\begin{array}{c}
x_{2} \\
-d x_{2}-g \sin x_{1}+u
\end{array}\right]
$$

where $\mathbf{x}=\left[x_{1}, x_{2}\right]^{T}=[q, \dot{q}]^{T}$ and $u=\tau$. In the following simulations, the damping coefficient $d$ is chosen as $d=0.01$. In the following simulations, the initial control sequence $u(t)$ used in ILQG is chosen to be zero.

1) Temporal Optimization: Using the techniques proposed in Section II-C, we consider the problem of optimizing the temporal aspect of the desired movement for a fixed set of parameters in the plant dynamics while ensuring the movement characteristics (e.g., amplitude) is maintained. The reference movement is given by the sinusoidal trajectory, $y(t)=0.5 \sin (\omega t)$, with the frequency $\omega$ to be optimized and the cost function is formulated as in (7) with $\mathbf{x}_{r e f}=$ 


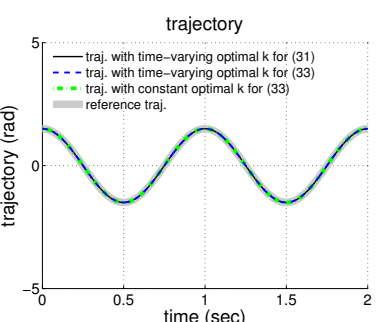

(a)

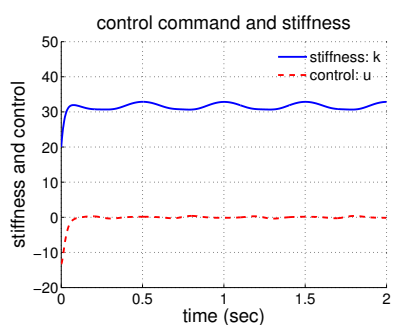

(b)

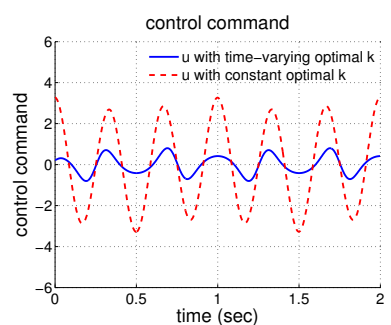

(c)

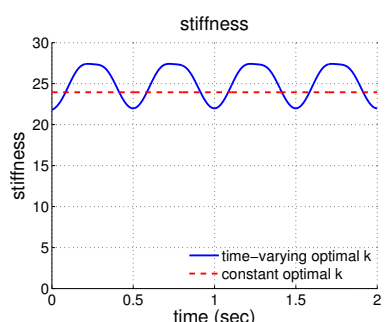

(d)

Fig. 3. Results of stiffness optimization: (a) reference trajectory and the joint trajectories. (b) optimized stiffness $k$ and control $u$ for the plant dynamics (29). Comparison of the (c) control $u$ (d) stiffness profiles for the plant dynamics (33) for time-varying and constant optimal stiffness profiles.

$[y, \dot{y}]^{T}$. We choose $\mathbf{Q}=\operatorname{diag}\{1000,1000\} \in \mathbb{R}^{2 \times 2}$, $\mathbf{R}=0.1 \in \mathbb{R}, \mathbf{P}_{T}=\mathbf{0} \in \mathbb{R}^{2 \times 2}$ in (8) and (9), respectively. ILQG and gradient descent with respect to $\omega$ are iteratively computed in order to obtain the optimal control law $u(x, t)$ in (10) and $\beta$ in (19) and in turn, optimal $\omega$.

Fig. 2 shows the comparison of the behavior with the initial $\omega=1.0$ (before optimization), during optimization steps of $\omega$ at 5, 10, 20 and 40 iterations, and the optimized $\omega=3.083$ (74 iterations). The results demonstrate a significant reduction in cost (from $J=35.598$ to $J=$ 0.001787 ) as result of requiring much smaller commands post optimization. The final optimized frequency $\omega=3.083$ showed a close match to the theoretically computed natural frequency $\omega_{0}=3.132$. This suggests that the proposed temporal optimization method is able to find an energy efficient temporal scaling of the movement pattern by exploiting the intrinsic dynamics of the plant. Notice that as demonstrated in this example, slow movement does not necessarily imply smaller control inputs.

2) Stiffness Optimization: Now consider the dynamics of the same pendulum (29) when driven by a variable stiffness actuator $\tau=-k q+u$ as in (22). The equation of motion is

$$
\ddot{q}+d \dot{q}+g \sin q=-k q+u .
$$

As discussed in Section II-D, in order to optimize the stiffness $k$ and obtain an optimal control law $u$ for a given periodic movement, the dynamics and control commands are augmented as

$$
\dot{\mathbf{x}}_{a u g}=\left[\begin{array}{c}
x_{2} \\
-d x_{2}-g \sin x_{1}+\left(-x_{3} x_{1}+u_{1}\right) \\
u_{2}
\end{array}\right]
$$

where $\mathbf{x}_{\text {aug }}=[\mathbf{x}, k]^{T}, \mathbf{x}=\left[x_{1}, x_{2}\right]^{T}=\left[\begin{array}{ll}q, & \dot{q}\end{array}\right]^{T}$ and $\mathbf{u}_{\text {aug }}=\left[u_{1}, u_{2}\right]^{T}=[u, \dot{k}]^{T}$. The reference joint trajectory is given by $y(t)=1.5 \sin (\omega t)$ with $\omega=2 \pi$. The weights of the cost function used are identical (see Section III-A) except $\mathbf{R}=\operatorname{diag}\{0.1,0.0001\} \in \mathbb{R}^{2 \times 2}$.

Fig. 3(b) shows the optimal control command $u$ and the time evolution of the optimized stiffness $k$. The stiffness is initialized as $k=20$ and it approximately approaches the desirable value $k=\omega^{2}-g=29.7$. Good tracking of the desired trajectory was achieved with visibly small error as can be seen in Fig. 3(a). Further, the effectiveness of the method is validated by comparing the total cost incurred with optimized time varying stiffness $(J=0.8130)$ as compared to the case with a constant stiffness of $k=20(J=83.348)$.
3) Time-varying Stiffness Optimization: In the above section, although we allowed for time-varying stiffness, since the system dynamics could be well characterized by its linear approximation, optimal solutions reflected nearly constant stiffness. However, in systems with strong non-linearities such as

$$
\ddot{q}+d \dot{q}+g \sin q+\bar{k} q^{3}=\tau
$$

where $\bar{k} q^{3}$ denotes a nonlinear spring, the concept of a natural or resonant frequency is more complicated. We perform an optimization using this modified plant with the same reference trajectory. Fig. 3(c) and (d) compare the results between time-varying optimal stiffness and constant optimal stiffness. In this comparison, constant optimal stiffness is obtained using a gradient descent update $k \leftarrow k-\alpha \nabla_{k} J$ and ILQG is used to obtain the control command iteratively. As shown in Fig.3(c), using time-varying optimal stiffness achieves more energy efficient control $(J=0.4421)$ requiring smaller control commands than using constant optimal stiffness $(J=2.219)$.

\section{B. Temporal Optimization in 2 DOF Underactuated System}

In this section, we apply the presented framework to the control of an underactuated system which has fewer actuators than the number of degrees of freedom. As an example plant, we consider a planar two-link robot arm under the influence of gravity where only the second joint has a control input (see Fig. 6 (left)), as seen in the Acrobot [23] and the brachiating robot [24], [25]—with characteristics similar to a gymnast on a high bar. The dynamics take a standard RBD form (11) with $\mathbf{q} \in \mathbb{R}^{2}$ and $\tau \in \mathbb{R}$ with no actuation on the first joint. Control of underactuated systems with passive joints is a challenging problem-this is because, while it is imperative to exploit system dynamics for task achievement, the control theory of such systems has not been well-established yet.

Specifically, we consider the tasks of (i) swing locomotion from handhold to handhold on a ladder (ii) swinging-up from the suspended posture by pumping up the energy in an appropriate manner to reach the target bar. The underactuation and the precise spatiotemporal nature of this statically unstable task-it is required to reach the target bar exactly at the correct time to grasp it; if the robot misses the bar, the body will swing backward due to the influence of gravity-makes this a challenging problem.

Note that the task objective is slightly different from the explicit trajectory tracking problems presented in the previous sections. In this example, the main objective is to show that it is possible to achieve highly dynamic and 

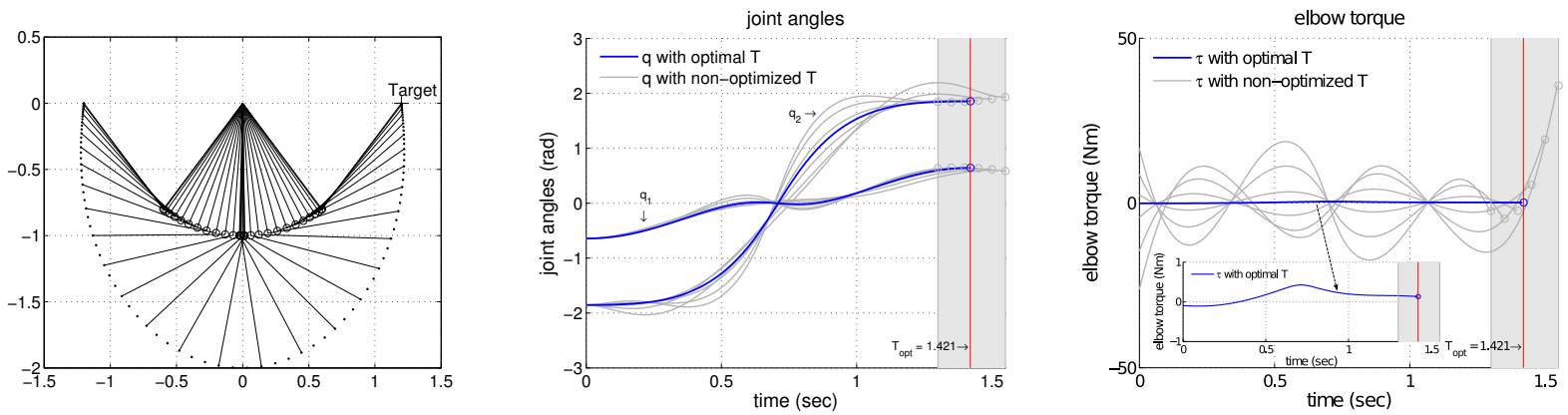

Fig. 4. Optimization of the locomotion task. Left: Movement of the robot. Center: Comparison of the joint angles between non-optimized $T$ in the range $T=[1.3, \ldots, 1.55](\mathrm{sec})$ and optimized $T=1.421(\mathrm{sec})$. Right: Comparison of the elbow torque command. Notice the significant reduction of the required elbow torque after time optimization although the joint trajectories look similar. $\tau$ only ranges between $[-0.1070 .425]$ during the movement with the optimized $T$ as shown in the embedded plot with a magnified range.

challenging maneuvers which require explicit exploitation of intrinsic dynamics of the system. We use the following cost function in both the locomotion and swing-up tasks considered here:

$$
J=\left(\mathbf{y}-\mathbf{y}^{*}\right)^{T} \mathbf{P}_{T}\left(\mathbf{y}-\mathbf{y}^{*}\right)+\int_{0}^{T} R u^{2} d t
$$

where $\mathbf{y}=[\mathbf{r}, \dot{\mathbf{r}}]^{T} \in \mathbb{R}^{4}$ is the position and velocity of the gripper in the Cartesian coordinates, $\mathbf{y}^{*}$ denotes its desired values when grasping, $\mathbf{P}_{T}$ is a positive definite matrix, $u$ is the elbow joint torque $u=\tau$ and $R$ is a positive scalar. In the following tasks, we consider temporal optimization to achieve energy efficient maneuvers to find the locally optimal duration of the movement in addition to finding the optimal control command $u$. We use the model of the robot (Fig. 6 (left)) with $m_{1}=1, m_{2}=3, l_{1}=l_{2}=l_{c 1}=l_{c 2}=1, I_{1}=$ $I_{2}=0$ and $d_{1}=d_{2}=0.05$. In the following simulations, the initial control sequence $u(t)$ used in ILQG is chosen to be zero.

1) Locomotion: Consider the task of moving from one to the next handhold by swinging the arms (from the left to right in Fig. 6). The distance between the handhold is dist $=1.2(\mathrm{~m})$. We optimized the duration of the movement $T$ and obtained the optimal control command $u$. Fig. 4 (left) depicts the optimized movement of the robot moving from the left to the right to grasp the target bar. Fig. 4 (center, right) overlays the joint trajectories and control commands of the fixed time horizon $T=1.3 \sim 1.55(\mathrm{sec})$ at the interval of 0.05 seconds and the optimized $T=1.421$ (sec). With the fixed time horizon, the cost ranged between $J=1.444$ with $T=1.4$ and $J=50.44$ with $T=1.3$ while the cost after temporal optimization was $J=0.00670$.

The joint trajectories of these movements look similar while we see significant difference in the required control commands within a narrow $0.25 \mathrm{sec}$ difference in movement duration. This highlights the sensitivity of such problems and reinforces the benefits that automated optimization techniques have over hand-tuned ones in fully exploiting the passive dynamics.

2) Swing-up Task: The swing-up task considers the movement from an initial suspended posture at rest with the aim to catch the next bar. This task is characterized by the necessity to move away from the desired goal in order to pump energy into the system combined with precise spatiotemporal
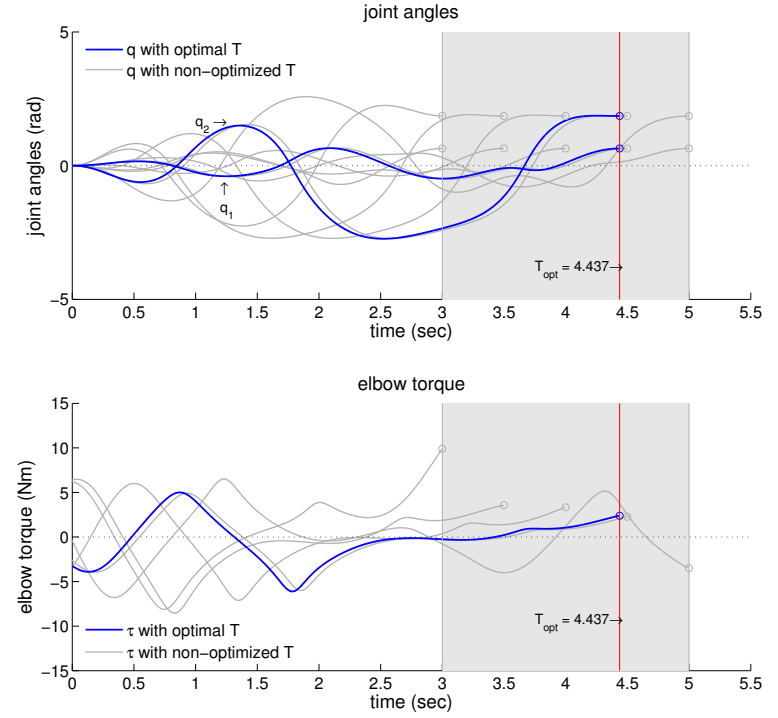

Fig. 5. Swing-up task: comparison of the joint angles (top) and elbow torque (bottom) between non-optimized $T=[3.0, \ldots, 4.5]$ (sec) and optimized $T=4.437$ (sec).

control for grasping. Here, we added an additional term to the cost that was linear in the movement duration $T$ as $J^{\prime}=J+w_{T} T$, where $J$ is the original cost function given in (34), and $w_{T}$ is a trade-off term that we choose as $w_{T}=10$. Fig. 6 (right) illustrates the obtained swing-up sequence of the robot followed by the locomotion behaviour. Fig. 5 shows the joint trajectories and elbow torque with fixed movement duration $T=[3.0,3.5,4.0,4.5,5.0]$ (sec) and with optimized movement duration $T=4.437$ (sec). Spatiotemporally optimized trajectories (lowest cost) were obtained, suggesting the suitability of the framework in even highly dynamic movements.

\section{CONCLUSION}

In this paper, we present a systematic method for stiffness and temporal optimization in periodic movements, with an emphasis on exploiting the intrinsic dynamics of the plant to realize efficient control. The proposed movement representation allows modulation of amplitude, frequency and offset of the movements while being particularly tuned 

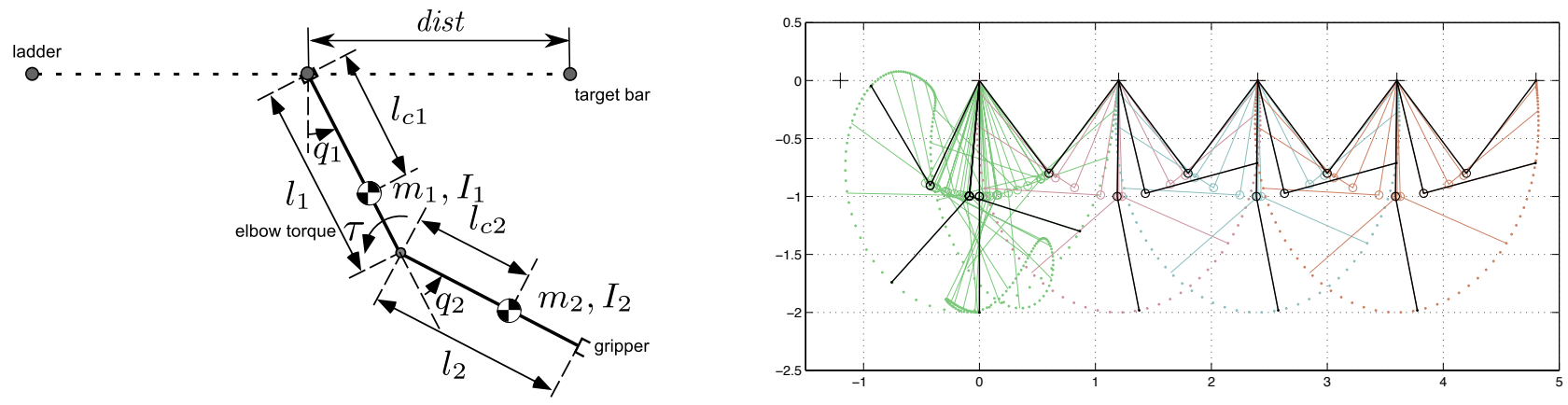

Fig. 6. (Left) A model of a two-link underactuated robot where only the second joint has a torque input $\tau$. (Right) Optimized swing-up and locomotion movement of the robot. The robot swings up from the suspended posture using the swing-up controller and moves towards the right by switching into the locomotion controller. In these maneuvers, the time-optimal control laws obtained in Sections III-B.1 and III-B.2 are used.

to the requirements of periodic or rhythmic movements. We formulate the optimization problem in the framework of optimal control and successively incorporate the ability to optimize for the control commands, temporal aspect of the movement as well as a time-varying stiffness profile. Numerical simulations demonstrate the effectiveness of the proposed approach in various tasks including stiffness and temporal optimization of oscillatory movements with a single pendulum, and locomotion and swing-up tasks of an underactuated brachiating 2 link robot. All the results highlight the significant reduction in control command magnitude (and costs) when using a spatiotemporally optimized actuation profile, demonstrating the ability of the framework to exploit the passive dynamics of the plant in a maximally beneficial way. In our future work, we will address optimization of periodic movements involving more complex trajectories, coordination of multiple degrees of freedom and hardware implementation on variable stiffness mechanisms in tasks such as walking, hopping or throwing. We would also like to explore the implication of the insights gained in this study on human gait analysis.

\section{ACKNOWLEDGMENTS}

This work was funded by the EU Seventh Framework Programme (FP7) as part of the STIFF project. We thank Matthew Howard, David Braun and Takeshi Mori for discussions in the SLMC group at the University of Edinburgh.

\section{REFERENCES}

[1] E. Todorov, "Optimality principles in sensorimotor control," Nature Neuroscience, vol. 7, no. 9, pp. 907-915, 2004.

[2] D. Mitrovic, S. Klanke, and S. Vijayakumar, "Learning Impedance Control of Antagonistic Systems Based on Stochastic Optimization Principles," Int. J. of Robotics Research, vol. 30, pp. 556-573, 2011.

[3] J. Morimoto and C. Atkeson, "Minimax differential dynamic programming: An application to robust biped walking," in NIPS, 2003.

[4] E. Westervelt, J. Grizzle, and D. Koditschek, "Hybrid zero dynamics of planar biped walkers," IEEE Trans. on Automatic Control, vol. 48, pp. 42-56, 2003.

[5] J. Morimoto and C. G. Atkeson, "Learning biped locomotion: Application of poincaré-map-based reinforcement learning," IEEE Robotics and Automation Magazine, vol. 14, pp. 41-51, 2007.

[6] I. Manchester, U. Mettin, F. Iida, and R. Tedrake, "Stable dynamic walking over uneven terrain," Int. J. of Robotics Research, vol. 30, pp. 265-279, 2011.

[7] J. Hench and A. Laub, "Numerical solution of the discrete-time periodic riccati equation," IEEE Trans. on Automatic Control, vol. 39, pp. 1197-1210, 1994.
[8] A. Shiriaev, L. Freidovich, and I. Manchester, "Can we make a robot ballerina perform a pirouette? orbital stabilization of periodic motions of underactuated mechanical systems," Annual Reviews in Control, vol. 32, pp. 200-211, 2008.

[9] A. Ijspeert, "Central pattern generators for locomotion control in animals and robots: A review," Neural Networks, vol. 21, pp. 642653, 2008.

[10] A. Ijspeert, J. Nakanishi, and S. Schaal, "Learning attractor landscapes for learning motor primitives," in NIPS, 2003

[11] W. $\mathrm{Li}$ and E. Todorov, "Iterative linearization methods for approximately optimal control and estimation of non-linear stochastic system," Int. J. of Control, vol. 80, pp. 1439-1453, 2007.

[12] K. Rawlik, M. Toussaint, and S. Vijayakumar, "An approximate inference approach to temporal optimization in optimal control," in NIPS, 2010

[13] Y. Ehara and S. Yamamoto, Introduction to Body-Dynamics-Analysis of Gait and Gait Initiation. Ishiyaku Publishers, 2002, in Japanese.

[14] D. Bennett, J. Hollerbach, Y. Xu, and I. Hunter, "Time-varying stiffness of human elbow joint during cyclic voluntary movement," Exp. Brain Res., vol. 88, pp. 433-442, 1992.

[15] M. Uemura and S. Kawamura, "Resonance-based motion control method for multi-joint robot through combining stiffness adaptation and iterative learning control," in IEEE Int. Conf. on Rob. and Aut., 2009

[16] G. Ganesh, A. Albu-Schaëffer, M. Haruno, M. Kawato, and E. Burdet, "Biomimetic motor behavior for simultaneous adaptation of force, impedance and trajectory in interaction tasks," in IEEE Int. Conf. on Rob. and Aut., 2010

[17] J. Buchli, E. Theodorou, F. Stulp, and S. Schaal, "Variable impedance control -a reinforcement learning approach," in Robotics: Science and Systems, 2010.

[18] F. Kuhl and C. Giardina, "Elliptic Fourier features of a closed contour," Computer Graphics and Image Processing, vol. 18, pp. 236-258, 1982.

[19] V. Arnold, Mathematical Methods of Classical Mechanics. SpringerVerlag, 1989.

[20] E. Theodorou, F. Valero-Cuevasu, and E. Todorov, "A first optimal control solution for a complex, nonlinear, tendon driven neuromuscular finger model," in ASME 2010 Summer Bioengineering Conf., 2010.

[21] M. Howard, D. Mitrovic, and S. Vijayakumar, "Transferring impedance control strategies between heterogeneous systems via apprenticeship learning," in IEEE-RAS Int. Conf. on Hum. Rob., 2010

[22] R. V. Ham, B. Vanderborght, M. Damme, B. Verrelst, and D. Lefeber, "Maccepa, the mechanically adjustable compliance and controllable equilibrium position actuator: Design and implementation in a biped robot," Robotics and Autonomous Systems, vol. 55, pp. 761-768, 2007.

[23] M. Spong, "The swing up control problem for the acrobot," IEEE Control Systems Magazine, vol. 15, pp. 49-55, 1995.

[24] F. Saito, T. Fukuda, and F. Arai, "Swing and locomotion control for a two-link brachiation robot," IEEE Control Systems Magazine, vol. 14, pp. 5-12, 1994.

[25] J. Nakanishi, T. Fukuda, and D. Koditschek, "A brachiating robot controller," IEEE Trans. on Robotics and Automation, vol. 16, pp. 109-123, 2000. 